This is the author's final, peer-reviewed manuscript as accepted for publication. The publisher-formatted version may be available through the publisher's web site or your institution's library.

\title{
On the DSM Newton-type method
}

\author{
A.G. Ramm
}

How to cite this manuscript

Ramm, A.G. (2012). On the DSM Newton-type method. Retrieved from http://krex.ksu.edu

\section{Published Version Information}

Citation: Ramm, A.G. (2012). On the DSM Newton-type method. Journal of Applied Mathematics and Computing, 38(1-2), 523-533.

Copyright: @ Korean Society for Computational and Applied Mathematics 2011

Digital Object Identifier (DOI): doi:10.1007/s12190-011-0494-z

Publisher's Link: http://www.springerlink.com/content/n246406280h7821k/

This item was retrieved from the K-State Research Exchange (K-REx), the institutional repository of Kansas State University. K-REx is available at http://krex.ksu.edu 
Journ. Appl. Math and Comp. (JAMC), 38, N1-2, (2012), 523-533. 


\title{
On the DSM Newton-type method
}

\author{
A. G. Ramm \\ Department of Mathematics \\ Kansas State University, Manhattan, KS 66506-2602, USA \\ ramm@math.ksu.edu
}

\begin{abstract}
A wide class of the operator equations $F(u)=h$ in a Hilbert space is studied. Convergence of a Dynamical Systems Method (DSM), based on the continuous analog of the Newton method, is proved without any smoothness assumptions on the $F^{\prime}(u)$. It is assumed that $F^{\prime}(u)$ depends on $u$ continuously. Existence and uniqueness of the solution to evolution equation $\dot{u}(t)=-\left[F^{\prime}(u(t))\right]^{-1}(F(u(t))-h), u(0)=u_{0}$, is proved without assuming that $F^{\prime}(u)$ satisfies the Lipschitz condition. The method of the proof is new. This method is based on a novel version of the abstract inverse function theorem.
\end{abstract}

MSC: 47J05, 47J07, 58C15; PACS 02.30.Hq; 02.30.Tb

Keywords: Inverse function theorem, Newton's method, DSM (Dynamical systems method), evolution equations

\section{Introduction}

In many cases one is interested in solving operator equation

$$
F(u)=h,
$$

where $F$ is a nonlinear operator in real Hilbert space $H$. Let us assume that equation (1.1) with $h=f$ has a solution $y$,

$$
F(y)=f,
$$

and that the Fréchet derivative $F^{\prime}(y)$ exists and is boundedly invertible:

$$
\left\|\left[F^{\prime}(y)\right]^{-1}\right\| \leq m, \quad m=\text { const }>0 .
$$

This assumption is relaxed in Remark 6, see Theorem 7, where the operator $\left[F^{\prime}(y)\right]^{-1}$ is unbounded and causes loss of smoothness: it acts similar to a differential operator, namely, from a Sobolev space $H_{a+b}$ into $H_{a}, b>0$. 
Equation (1.2) was studied in many books and papers, see [1-4], because a wide range of problems can be reduced to solving this equation.

Let us also assume that $F^{\prime}(u)$ exists in the ball $B(y, R):=\{u:\|u-y\| \leq R\}$, depends continuously on $u$, and $\omega(R)$ is its modulus of continuity in the ball $B(y, R)$ :

$$
\sup _{u, v \in B(y, R),\|u-v\| \leq r}\left\|F^{\prime}(u)-F^{\prime}(v)\right\|=\omega(r) .
$$

The function $\omega(r) \geq 0$ is assumed to be continuous on the interval $[0,2 R]$, strictly increasing, and $\omega(0)=0$. Assumption (1.4) is much weaker than the usual Lipschitz condition on $F^{\prime}(u)$. The Lipschitz condition implies that $\omega(r)=$ $L r$ for some constant $L>0$. Assumption (1.4) means that $\omega(r)$ is just a continuous strictly increasing function, and $\omega(0)=0$. For example, one may have $\omega(r)=c r^{a}$, where $c=$ const $>0$ and $a>0$ is an arbitrary small number.

A widely used method for solving equation (1.1) is the Newton method:

$$
u_{n+1}=u_{n}-\left[F^{\prime}\left(u_{n}\right)\right]^{-1} F\left(u_{n}\right), \quad u_{0}=z,
$$

where $z$ is an initial approximation. Sufficient condition for the convergence of the iterative scheme 1.5 to the solution $y$ of equation 1.1 are proposed in [1-[5], and in references therein. These conditions in most cases require a Lipschitz condition for $F^{\prime}(u)$, a sufficient closeness of the initial approximation $u_{0}$ to the solution $y$, and other conditions ( see, for example, [1, p.157).

In 5], [6],[9]-[11], [14]-[15], a general method, the Dynamical Systems Method (DSM), is developed for solving equation 1.2 .

This method consists of finding a nonlinear operator $\Phi(t, u)$ such that the Cauchy problem

$$
\dot{u}=\Phi(t, u), \quad u(0)=u_{0},
$$

has a unique global solution $u=u\left(t ; u_{0}\right)$, there exists $u(\infty)=\lim _{t \rightarrow \infty} u\left(t ; u_{0}\right)$, and $F(u(\infty))=f$ :

$$
\exists ! u(t), \quad \forall t \geq 0 ; \quad \exists u(\infty) ; \quad F(u(\infty))=f .
$$

Many examples of the possible choices of $\Phi(t, u)$ are given in [5. Theoretical applications of the DSM are proposed in [7, 8]. A particular choice of $\Phi$, namely, $\Phi=-\left[F^{\prime}(u)\right]^{-1}(F(u)-h)$, leads to a continuous analog of the Newton method:

$$
\dot{u}(t)=-\left[F^{\prime}(u(t))\right]^{-1}(F(u(t))-h), \quad u(0)=u_{0} ; \quad \dot{u}(t)=\frac{d u(t)}{d t},
$$

for solving equation (1.1).

The question of general interest is:

Under what assumptions on $F, h$ and $u_{0}$, can one establish the conclusions (1.7), that is, the global existence and uniqueness of the solution to problem (1.8), the existence of $u(\infty)$, and the relation $F(u(\infty))=h$ ?

The usual condition, sufficient for the local existence and uniqueness of the solution to (1.8), is the local Lipschitz condition on the right-hand side of (1.8). 
Such condition can be satisfied, in general, only if $F^{\prime}(u)$ satisfies a Lipschitz condition.

Our goal is to develop a novel approach to a study of equation (1.8). This approach does not require a Lipschitz condition for $F^{\prime}(u)$, and it leads to a justification of the conclusion (1.7) (with $h$ replacing $f$ ) for the solution to problem (1.8) under natural assumptions on $h$ and $u_{0}$. In [12, such justification are given for $F$ which are locally $C^{1}$-smooth global homeomorphisms, and in [13] this justification is given for $F$ satisfying some spectral assumptions in Banach spaces.

We prove convergence of the continuous analog (1.8) of the Newton method without any smoothness assumptions on $F^{\prime}(u)$, only the local continuity of $F^{\prime}(u)$ is assumed, see (1.4).

This approach uses the special structure of equation (1.8), which corresponds to the Newton-type methods. The Newton-type methods are widely used in theoretical, numerical and applied research, and by this reason our results are of general interest.

Our results demonstrate the universality of the Newton-type methods in the following sense: we prove that any operator equation 1.1 can be solved by the DSM Newton method (1.8), provided that conditions (1.3)- 1.4 hold, the initial approximation $u_{0}$ is sufficiently close to $y$, where $y$ is the solution of equation (1.2), the right-hand side $h$ in 1.1 is sufficiently close to $f$, and equation 1.2 with the right-hand side $f$ is solvable.

Precise formulations of the results are given in eight theorems.

The basic tool in this paper is a new version of the inverse function theorem. The method of the proof of this theorem is quite similar to the method of the proof of the standard inverse function theorem.

The novelty of our version of the inverse function theorem is in a specification of the region in which the abstract inverse function exists.

This is done in terms of the modulus of continuity of the operator $F^{\prime}(u)$ in the ball $B(y, R)$.

In Section 2 we formulate and prove this version of the inverse function theorem. The result is stated as Theorem 1 .

In Section 3 we justify the DSM for equation (1.8). The result is stated in Theorem 3. Moreover, we generalize the result to the case when assumption (1.3) is not valid, and the operator $\left[F^{\prime}(u)\right]^{-1}$ is unbounded, acting similar to a differential operator and causing the "loss of derivatives". The result is stated in Theorem 7 .

In Section 4 we prove convergence of the usual Newton method 1.5 . The result is stated in Theorem 8 .

\section{Inverse function theorem}

Consider equation (1.1). Let us make the following assumptions:

Assumptions A):

A1): Equation (1.2) and estimates (1.3), 1.4 hold in $B(y, R)$, 
A2): $h \in B(f, \rho), \quad \rho=\frac{(1-q) R}{m}, \quad q \in(0,1)$,

A3): $m \omega(R)=q, \quad q \in(0,1)$.

Assumption A3) defines $R$ uniquely because $\omega(r)$ is strictly increasing. We assume that equation $m \omega(R)=q$ has a solution. This assumption is always satisfied if $q \in(0,1)$ is sufficiently small. The constant $m$ is defined in (1.3). The radius $R$ can be defined by the formula $R=\omega^{-1}\left(q m^{-1}\right)$, where $\omega^{-1}$ is the inverse function. Since $\omega(r)$ is strictly increasing, its inverse function $\omega^{-1}$ is well defined. The number $q$ in the second condition of Assumptions $A$ ) is the same as in the third condition. The number $q$ can be fixed in the interval $(0,1)$, and then the number $R>0$ is uniquely found from the equation $\omega(R)=q m^{-1}$, because $\omega(R)$ is strictly growing and $\omega(0)=0$. The number $q$ may depend on $f$, because $y$ depends on $f$, and the radius $R$ of a ball $B(y, R)$ in which $\omega(r)$ is continuous for a given operator $F^{\prime}(u)$ may depend on $y$.

Our first result, Theorem 1, says that under Assumptions A) equation (1.1) is uniquely solvable for any $h$ in a sufficiently small neighborhood of $f$.

Theorem 1. If Assumptions A1)-A3) hold, then equation (1.1) has a unique solution $u$ for any $h \in B(f, \rho)$, and

$$
\left\|\left[F^{\prime}(u)\right]^{-1}\right\| \leq \frac{m}{1-q}, \quad \forall u \in B(y, R) .
$$

Proof. Let us denote

$$
Q:=\left[F^{\prime}(y)\right]^{-1}, \quad\|Q\| \leq m
$$

Then equation (1.1) is equivalent to

$$
u=T(u), \quad T(u):=u-Q(F(u)-h) .
$$

Let us check that $T$ maps the ball $B(y, R)$ into itself:

$$
T B(y, R) \subset B(y, R),
$$

and that $T$ is a contraction mapping in this ball:

$$
\|T(u)-T(v)\| \leq q\|u-v\|, \quad \forall u, v \in B(y, R),
$$

where $q \in(0,1)$ is defined in Assumptions $A)$.

If $(2.2)$ and $(2.3)$ are verified, then the contraction mapping principle guarantees existence and uniqueness of the solution to equation 2.2 in $B(y, R)$, where $R$ is defined by condition 3) in Assumptions A).

Let us check the inclusion 2.3 . One has

$$
J_{1}:=\|u-y-Q(F(u)-h)\|=\|u-y-Q[F(u)-F(y)+f-h]\|,
$$

and

$$
\begin{aligned}
F(u)-F(y) & =\int_{0}^{1} F^{\prime}(y+s(u-y)) d s(u-y) \\
& =F^{\prime}(y)(u-y)+\int_{0}^{1}\left[F^{\prime}(y+s(u-y))-F^{\prime}(y)\right] d s(u-y) .
\end{aligned}
$$


Note that

$$
\|Q(f-h)\| \leq m \rho,
$$

and

$$
\sup _{s \in[0,1]}\left\|F^{\prime}(y+s(u-y))-F^{\prime}(y)\right\| \leq \omega(R) .
$$

Therefore, for any $u \in B(y, R)$ one gets from (1.3), 2.4) and 2.5 the following estimate:

$$
J_{1} \leq m \rho+m \omega(R) R \leq(1-q) R+q R=R,
$$

where the inequalities

$$
\|f-h\| \leq \rho, \quad\|u-y\| \leq R,
$$

and assumptions A2) and A3) in Assumptions A) were used.

Let us establish inequality (2.4):

$$
\begin{gathered}
J_{2}:=\|T(u)-T(v)\|=\|u-v-Q(F(u)-F(v))\| \\
F(u)-F(v)=F^{\prime}(y)(u-v)+\int_{0}^{1}\left[F^{\prime}(v+s(u-v))-F^{\prime}(y)\right] d s(u-v) .
\end{gathered}
$$

Note that

$$
\|v+s(u-v)-y\|=\|(1-s)(v-y)+s(u-y)\| \leq(1-s) R+s R=R .
$$

Thus, from $(2.9)$ and 2.10$)$ one gets

$$
J_{2} \leq m \omega(R)\|u-v\| \leq q\|u-v\|, \quad \forall u, v \in B(y, R) .
$$

Therefore, both conditions (2.3) and (2.4) are verified. Consequently, the existence of the unique solution to 1.1 in $B(y, R)$ is proved.

Let us prove estimate (2.1). One has

$$
\begin{aligned}
{\left[F^{\prime}(u)\right]^{-1} } & =\left[F^{\prime}(y)+F^{\prime}(u)-F^{\prime}(y)\right]^{-1} \\
& =\left[I+\left(F^{\prime}(y)\right)^{-1}\left(F^{\prime}(u)-F^{\prime}(y)\right)\right]^{-1}\left[F^{\prime}(y)\right]^{-1},
\end{aligned}
$$

and

$$
\left\|\left(F^{\prime}(y)\right)^{-1}\left(F^{\prime}(u)-F^{\prime}(y)\right)\right\| \leq m \omega(R) \leq q, \quad u \in B(y, R) .
$$

It is well known that if a linear operator $A$ satisfies the estimate $\|A\| \leq q$, where $q \in(0,1)$, then the inverse operator $(I+A)^{-1}$ does exist, and ||$(I+A)^{-1} \mid \leq \frac{1}{1-q}$. Thus, the operator $\left[I+\left(F^{\prime}(y)\right)^{-1}\left(F^{\prime}(u)-F^{\prime}(y)\right)\right]^{-1}$ exists and its norm can be estimated as follows:

$$
\left\|\left[I+\left(F^{\prime}(y)\right)^{-1}\left(F^{\prime}(u)-F^{\prime}(y)\right)\right]^{-1}\right\| \leq \frac{1}{1-q} .
$$

Consequently, (2.12) and (2.14) imply (2.1). Thus, estimate $(2.1)$ is proved.

Theorem 1 is proved. 
Remark 2. If $h=h(t) \in C^{1}([0, T])$, then the solution $u=u(t)$ of equation (1.1) is $C^{1}([0, T])$ provided that Assumptions A) hold.

Indeed, if $h=h(t)$, then a formal differentiation of equation 1.1) with respect to $t$ yields:

$$
F^{\prime}(u(t)) \dot{u}(t)=\dot{h}(t) .
$$

Since $u(t) \in B(y, R)$, the operator $F^{\prime}(u(t))$ is boundedly invertible and depends continuously on $t$ because $u(t)$ does. Thus,

$$
\dot{u}(t)=\left[F^{\prime}(u(t))\right]^{-1} \dot{h}(t),
$$

so $\dot{u}(t)$ depends on $t$ continuously.

The formal differentiation is justified if one proves that $u(t)$ is differentiable at any $t \in[0, T]$, that is,

$$
u(t+k)-u(t)=A(t) k+o(k), \quad k \rightarrow 0, \quad t \in[0, T],
$$

where $A(t) \in H$ does not depend on $k$ and at the ends of the interval $[0, T]$ the derivatives are understood as one-sided.

To establish relation (2.16) one uses equation (1.1) and the assumption $h \in$ $C^{1}([0, T])$. One has:

$$
F(u(t+k))-F(u(t))=h(t+k)-h(t)=\dot{h}(t) k+o(k), \quad k \rightarrow 0,
$$

and

$$
F(u(t+k))-F(u(t))=\int_{0}^{1} F^{\prime}(u(t)+s(u(t+k)-u(t))) d s(u(t+k)-u(t)) .
$$

The operator $\int_{0}^{1} F^{\prime}(u(t)+s[u(t+k)-u(t)]) d s$ is boundedly invertible (uniformly with respect to $k \in\left(0, k_{0}\right)$, where $0<k_{0}$ is a sufficiently small number) as long as

$$
\sup _{s \in[0,1]}\|u(t)+s(u(t+k)-u(t))-y\| \leq R,
$$

see (2.1). This inequality holds, as one can easily check:

$\|u(t)+s(u(t+k)-u(t))-y\|=\|(1-s)(u(t)-y)+s(u(t+k)-y)\| \leq(1-s) R+s R=R$.

Therefore, 2.16 follows from (2.17) and 2.18). Remark 2 is proved.

\section{Convergence of the DSM (1.8)}

Consider the following equation

$$
F(u)=h+v(t)
$$

where

$$
u=u(t), \quad v(t)=e^{-t} v_{0}, \quad v_{0}:=F\left(u_{0}\right)-h, \quad r=\left\|v_{0}\right\| .
$$


At $t=0$ equation (3.1) has a unique solution $u_{0}$.

Let us make the following Assumptions B):

B1): Assumptions A) hold,

B2): $h \in B(f, \delta), \quad \delta+r \leq \rho:=\frac{(1-q) R}{m}$.

Let us recall that our assumptions are much weaker than the Lipschitz assumption on $F^{\prime}(u)$. For example, if $\left\|F^{\prime}(u)-F^{\prime}(v)\right\| \leq c\|u-v\|^{a}$, where $c>0$ and $a>0$ are constants, and $a<1$, then the Lipschitz condition for $F^{\prime}(u)$ is not satisfied, but our assumptions on $\omega(r)$ are satisfied. The assumptions concerning $\omega(r)$ are easier to check than the Lipschitz assumption on $F^{\prime}(u)$, as the above example shows.

Theorem 3. If Assumptions B1)-B2) hold, then conclusions (1.7), with $f$ replaced by $h$, hold for the solution of problem 1.8.

Proof. The proof is divided into three parts.

Part 1. Proof of the global existence and uniqueness of the solution to problem 1.8 .

One has

$$
\|h+v(t)-f\| \leq\|h-f\|+\left\|v_{0} e^{-t}\right\| \leq \delta+r \leq \rho, \quad \forall t \geq 0 .
$$

Thus, it follows from Theorem 1 that equation (3.1) has a unique solution

$$
u=u(t) \in B(y, R)
$$

$\forall t \in[0, \infty)$. Moreover, $u(t) \in C^{1}([0, \infty))$ by Remark 2 .

Differentiation of (3.1) with respect to $t$ yields

$$
F^{\prime}(u) \dot{u}=\dot{v}=-v=-(F(u(t))-h) .
$$

Since $u(t) \in B(y, R)$, the operator $F^{\prime}(u(t))$ is boundedly invertible, so equation (3.3) is equivalent to (1.8). The initial condition $u(0)=u_{0}$ is satisfied, as was mentioned below $(3.2)$. Therefore, the existence of the unique global solution to 1.8 is proved.

Part 2. Proof of the existence of $u(\infty)$.

From (3.1), (3.2), 2.1), and (1.8) it follows that

$$
\|\dot{u}\| \leq \frac{m r}{1-q} e^{-t}, \quad q \in(0,1) .
$$

This and the Cauchy criterion for the existence of the limit $u(\infty)$ imply that $u(\infty)$ exists.

Integrating (3.4), one gets

$$
\left\|u(t)-u_{0}\right\| \leq \frac{m r}{1-q},
$$

and

$$
\|u(\infty)-u(t)\| \leq \frac{m r}{1-q} e^{-t}
$$


Part 3. Proof of the relation $F(u(\infty))=h$.

The desired relation follows from (3.1) and (3.2) as $t \rightarrow \infty$, because $v(\infty)=$ $0, u(t) \in B(y, R)$, and $F$ is continuous in $B(y, R)$.

Theorem 3 is proved.

Remark 4. Let us explain why there is no assumption on the location of $u_{0}$ in Theorem 3. The reason is simple: in the proof of Theorem 3 it was established that $u(t) \in B(y, R)$ for all $t \geq 0$. Therefore, it follows that the assumptions of Theorem 3 imply that $u_{0}=u(0) \in B(y, R)$.

From the proof of Theorem 3 one obtains the following result.

Theorem 5. Assume that $F$ is a global homeomorphism, that $\left\|\left[F^{\prime}(u)\right]^{-1}\right\| \leq$ $m(u)$, where $m(u)>0$ is a constant which depends on $u$, and that $F^{\prime}(u)$ is continuous with respect to $u$. Then problem 1.8 has a unique global solution for any $h$ and any $u_{0}$, there exists $u(\infty)$, and $F(u(\infty))=h$.

Proof. If $F$ is a global homeomorphism, then equation 3.1 is uniquely solvable for any $v(t)$. Differentiation of this equation with respect to $t$ yields equation (3.3), and this equation is equivalent to (1.8) because of the bounded invertibility of $F^{\prime}(u)$ at any $u$. The existence of $u(\infty)$ and the equality $F(u(\infty))=h$ follow from the relation $\lim _{t \rightarrow \infty}(h+v(t))=h$ and from the assumption that $F$ is a global homeomorphism. Theorem 5 is proved.

A practically important example of equations (1.1) with a global homeomorphism $F$ is the equation

$$
F(u):=G(u)+b u=h,
$$

where $b=$ const $>0$ and $G$ is a monotone Fréchet differentiable operator. One has $F^{\prime}(u)=G^{\prime}(u)+b I$ and $\left\|\left[G^{\prime}(u)+b I\right]^{-1}\right\| \leq \frac{1}{b}$, because the monotonicity of $G$ implies $G^{\prime}(u) \geq 0$. Recall that if a linear operator $A \geq 0$, and $b=$ const $>0$, then $\left\|(A+b I)^{-1}\right\| \leq \frac{1}{b}$.

It is known that such $F$ are global homeomorphisms (see, e.g., [1]). For such $F$ equation (1.1) can be solved for any $h$ by the DSM Newton method (1.8) with any initial approximation $u_{0}$. In this sense convergence of the DSM Newton method (1.8) is global for the $F$, satisfying the above assumptions.

Remark 6. Our arguments are generalized to the case when $A:=F^{\prime}(u)$ acts from $H=H_{a}$ into $H_{a+b} \subset H$, so $A$ is a smoothing operator, and its inverse $\left[F^{\prime}(u)\right]^{-1}$ acts as a differential operator, so $A^{-1}$ is unbounded in $H$. For example, let $H_{a}$ be a Hilbert scale of spaces, $H_{a} \subset H_{b}$ if $a>b \geq 0$. A typical example is the case of Sobolev spaces $H_{a}, H_{0}:=H=L^{2}(D)$.

Let us assume that the following Assumptions $C$ ) hold:

C1): $\quad F: H_{a} \rightarrow H_{a+b}, b>0$;

the Fréchet derivative $A(u):=F^{\prime}(u): H_{a} \rightarrow H_{a+b}$ exists and is continuous with respect to $u$ in the following sense:

$$
\|A(u)-A(v)\|_{a+b} \leq \omega\left(\|u-v\|_{a}\right), \quad \forall u, v \in B(y, R),
$$


where $y$ solves the equation $F(y)=f$, the function $\omega(r)$ is continuous and strictly increasing for $r \in\left(0, R_{1}\right), \omega(r) \geq 0, \omega(0)=0, R_{1}>0$ is a sufficiently large constant, so that equation

$$
m \omega(R)=q, \quad q \in(0,1),
$$

has a unique solution $R<R_{1}$, and $m>0$ is a constant in the following inequality:

$$
\left\|[A(y)]^{-1} g\right\|_{a} \leq m\|g\|_{a+b}, \quad \forall g \in H_{a+b},
$$

C2): $\quad h \in B(f, \rho)$, where $\rho=\frac{(1-q) R}{m}$.

Theorem 7. If Assumptions C1)-C2) hold, then for any $h \in B(f, \rho)$ there exists a unique $u \in B(y, R)$ such that $F(u)=h$. The operator $F^{-1}$ is continuous on $B(f, \rho)$.

Proof. The proof is similar to the proof of Theorem 1.

This Theorem deals with the case when the operator $A^{-1}(u)$ causes loss of $b>0$ derivatives, it acts similarly to a differentiation operator of order $b>0$.

One can prove that conclusions (1.7) hold for (1.8) if Assumptions C1)-C2) hold and $h \in B(f, \delta)$, where $\delta+r \leq \rho, r=\left\|F\left(u_{0}\right)-h\right\|_{a+b}$, and $\rho$ is the same as in Assumptions (1)-C2). The proof is similar to the proof of Theorem 3.

\section{The Newton method}

The goal in this Section is to prove convergence of the Newton method

$$
u_{n+1}=u_{n}-\left[F^{\prime}\left(u_{n}\right)\right]^{-1}\left(F\left(u_{n}\right)-f\right), \quad u_{0}=z,
$$

to the solution $y$ of equation $(1.2)$ without any additional assumptions on the smoothness of $F^{\prime}(u)$. Only the continuity of $F^{\prime}(u)$ with respect to $u \in B(y, R)$ is assumed. The notations are the same as in Assumptions A) in Section 2. By $z \in H$ we denote an initial approximation.

Theorem 8. Assume that (1.2)-(1.4) and Assumptions A) hold, and that

$$
m \omega(R)=q \in\left(0, \frac{1}{2}\right), \quad q_{1}\|z-y\| \leq R, \quad q_{1}:=\frac{q}{1-q} .
$$

Then process (4.1) converges to $y$.

Proof. One has

$$
\begin{aligned}
u_{n+1}-y & =u_{n}-y-\left[F^{\prime}\left(u_{n}\right)\right]^{-1} \int_{0}^{1} F^{\prime}\left(y+s\left(u_{n}-y\right)\right) d s\left(u_{n}-y\right) \\
& =-\left[F^{\prime}\left(u_{n}\right)\right]^{-1} \int_{0}^{1}\left[F^{\prime}\left(y+s\left(u_{n}-y\right)\right)-F^{\prime}\left(u_{n}\right)\right] d s\left(u_{n}-y\right)
\end{aligned}
$$


Let

$$
a_{n}:=\left\|u_{n}-y\right\|, \quad a_{0}=\|z-y\| .
$$

Then 4.2, 4.3) and 2.1) imply

$$
a_{n+1} \leq \frac{m \omega(R)}{1-q} a_{n} \leq \frac{q}{1-q} a_{n}:=q_{1} a_{n} .
$$

From the assumption $q \in\left(0, \frac{1}{2}\right)$ one derives that $q_{1} \in(0,1)$. Thus, using 4.27 , one gets:

$$
\left\|u_{1}-y\right\|:=a_{1} \leq q_{1} a_{0} \leq R .
$$

By induction one obtains:

$$
\left\|u_{n}-y\right\| \leq R, \quad \forall n=1,2,3, \ldots .
$$

Consequently, $u_{n} \in B(y, R)$ for all $n$, and estimates (2.1) and (4.4) are applicable for all $n$. Therefore, (4.4) implies

$$
a_{n} \leq q_{1}^{n-1} R, \quad \forall n=1,2,3, \ldots \ldots .
$$

Thus,

$$
\lim _{n \rightarrow \infty} a_{n}=0 .
$$

Theorem 8 is proved.

Remark 9. It is of interest to find out if the global convergence of the Newton method (4.1) holds under the assumptions of Theorem 5. By global convergence we mean convergence for any $f$ and any $z$. This question is currently open. Therefore, the DSM is easier to study than its discrete analog (4.1). Since the global convergence of the DSM is proved under the assumptions of Theorem 5, one may conjecture that its discrete analog (4.1) also convereges globally. 


\section{References}

[1] K. Deimling, Nonlinear functional analysis, Springer-Verlag, Berlin, 1985.

[2] L. Kantorovich, G. Akilov, Functional analysis, Pergamon Press, New York, 1982.

[3] M. Krasnosel'skii et al, Approximate solutions of operator equations, Groningen, Noordhoff, 1972.

[4] J. Ortega, W. Rheinboldt, Iterative solution of nonlinear equations in several variables, SIAM, Philadelphia, 2000.

[5] A. G. Ramm, Dynamical systems method for solving operator equations, Elsevier, Amsterdam, 2007.

[6] A. G. Ramm, Inverse problems, Springer, New York, 2005.

[7] A. G. Ramm, Dynamical systems method and a homeomorphism theorem, Amer. Math. Monthly, 113, N10, (2006), 928-933.

[8] A. G. Ramm, Implicit function theorem via the DSM, Nonlinear Analysis: Theory, Methods and Appl., 72, N3-4, (2010), 1916-1921.

[9] A. G. Ramm, Dynamical systems method for solving operator equations, Communic. in Nonlinear Sci. and Numer. Simulation, 9, N2, (2004), 383402.

[10] A. G. Ramm, Dynamical systems method (DSM) and nonlinear problems, in the book: Spectral Theory and Nonlinear Analysis, World Scientific Publishers, Singapore, 2005, 201-228. (ed J. Lopez-Gomez).

[11] A. G. Ramm, Global convergence for ill-posed equations with monotone operators: the dynamical systems method, J. Phys A, 36, (2003), L249L254.

[12] A. G. Ramm, Justification of the Dynamical Systems Method (DSM) for global homeomorphisms, Eurasian Math. Journ., 1(4), 116-123, (2010)

[13] A. G. Ramm, How large is the class of operator equations solvable by a DSM Newton-type method? Appl. Math. Lett., 24(6), 860-865, (2011).

[14] N.S.Hoang and A. G. Ramm, DSM of Newton-type for solving operator equations $F(u)=f$ with minimal smoothness assumptions on $F$, International Journ. Comp.Sci. and Math. (IJCSM), 3 (1/2), 3-55, (2010).

[15] N.S.Hoang and A. G. Ramm, The Dynamical Systems Method for solving nonlinear equations with monotone operators, Asian Europ. Math. Journ., $3(1), 57-105,(2010)$. 